Cuadernos de Filología Italiana

ISSN: $1133-9527$

http://dx.doi.org/10.5209/CFIT.58805

\title{
Primi appunti sulla lessicografia piemontese dell'Ottocento*
}

Manuel Barbera ${ }^{1}$

Ricevuto 05 febbraio 2018 / Modificato: 30 maggio 2018 / Accettato: 12 giugno 2018

Riassunto. Si delineano brevemente le linee maestre della lessicografia del piemontese, con i suoi precedenti ed immediati conseguenti, caratterizzandone le vari fasi in cui si scandisce.

Parole chiave: Piemontese; Lessicografia; Ottocento; Storia della linguistica.

\section{[en] First notes on Piedmontese lexicography in the Nineteenth century}

Abstract. The main lines of Piedmontese lexicography are briefly outlined, with its precedents and consequences. Its various phases are explained and characterized.

Keywords. Piedmontese; Lexicography; Nineteenth century; History of linguistics

Sommario: 1. Premessa 2. Prodromi 3. Capello e Zalli 4. Il Ponza. 5. Il Sant'Albino. 6. Pasquali e Gavuzzi. 7. Epilegomena 8. Conclusioni 9. Appendice: i testi.

Come citare: Barbera, Manuel (2018) «Primi appunti sulla lessicografia piemontese dell'Ottocento», Cuadernos de Filología Italiana, 25, pp. 11-36.

\section{Premessa}

La tradizione lessicografica piemontese, anche escludendo la moderna, vanta una tradizione ed una ricchezza che poche "lingue minori" d'Italia possono vantare. I suoi capisaldi², infatti, possono condensarsi in:

$$
\begin{aligned}
& \text { Vopisco }(1564)-\text { Pipino }(1783)-\text { Capello }(1814)-\text { Zalli }(1815)-(1830)- \\
& \text { Ponza }(1826)-(1827)-(1830-33)-(1834)-(1843)-(1844)-(1845) \\
& \text { - }(1847)-(1859)-(1860)-\text { Sant'Albino }(1859)-\text { Pasquali }(1869)- \\
& (1870)-\text { Gavuzzi }(1891)-(1896)
\end{aligned}
$$

\footnotetext{
* Nell'introdurre queste mie note, mi soccorre ringraziare Luca Bellone, Massimo Fanfani, Carla Marello, Giovanni Ronco e soprattutto mio padre, grande conoscitore di libri e cose piemontesi, cui il presente lavoro è dedicato. Naturalmente di tutte le colpe, certo non poche, di cui si sarà inevitabilmente macchiato, è responsabile solo l'autore.

1 Università degli studi di Torino, Via Piazzi 58 - 10129 Torino. b.manuel@inrete.it.

2 Per gli episodi “minori” si veda invece la bibliografia dei Clivio.
} 


\section{Cui eventualmente potrebbero aggiungersi}

$$
\text { D’Azeglio (1886) — Rosa (1889) — Viriglio (1897) — (1917) — Comune (1923) }
$$

$\mathrm{Su}$ questa tradizione ha di recente portato attenzione ${ }^{3}$ ''attesissima pubblicazione del REP; l'interesse (più che comprensibile) del REP era però soprattutto sulla consistenza dei lemmari, e forse qualche altra considerazione storiografica, in parte più lessicografica, merita di esservi aggiunta. Concentrandoci sull'Ottocento, e tralasciando l'etimologia ${ }^{4}$, accenneremo pertanto uno schizzo di detta traiettoria; ulteriori aspetti saranno oggetto di altri interventi.

Si tratta, in effetti, di una tradizione insolitamente ricca nel panorama lessicografico dei volgari d'Italia, che difficilmente vantano una così copiosa produzione di dizionari ${ }^{5}$.

\section{Prodromi}

Il settecentesco dizionario del medico Maurizio Pipino si può considerare a tutti gli effetti il primo dizionario piemontese, anche se invero è stato preceduto nel Cinquecento $^{6}$ dal «casuale episodio» del Prontuario del Vopisco ${ }^{7}$.

\subsection{Il Vopisco}

«Casuale», per usare le parole della Cornagliotti (1991: 313), che così le commenta: «dico casuale perché la data è da considerarsi assai antica per un dizionario dialettale, perché il Vopisco non era nativo del Piemonte ma della Campania (ha infatti presente l'opera dello Scoppa), perché i destinatari, gli allievi delle scuole monregalesi, costituiscono un pubblico scelto e infine perché il piemontese riprodotto, ossia la varietà sud-occidentale, può presentare segni di infiltrazione provenzale oltre che francese».

3 In realtà un paio di precedenti ci sono: L. Ramello (2004), che però, per quanto meritorio e fondante, non ha ancora vere mire di storiografia linguistica, ma è descrittivo-informativo, ed è piuttosto finalizzato alla contemporaneità: infatti giunge a tutto il '900, ma esclude, ad esempio, il Viriglio ed analoghi; e Telmon (2015).

4 Anche se qualche piccolo sconfinamento si renderà necessario per il Pasquali e per il Rosa. L'esclusione è per varie ragioni: vuoi perché dopo il REP è compito superfluo, vuoi perché costituisce un settore sui generis, molto speciale ed individuato, e vuoi perché prende piede solo nella fase terminale della parabola qui considerata: gli apripista sono infatti il ben poco scientifico Glossario del Dal Pozzo (1888, di c. 3.000 voci), e l'invece saldo ancorché breve studio sui germanismi (1883) del Rosa. Analogo comportamento terremo per la lessicografia di àmbito specialistico, di cui la CB: 184-95 offre comunque un elenco esauriente specie per i settori botanici e zoologici.

5 Che certo, soprattutto per l'Ottocento, è anche portato dal ruolo politico giocato dallo Stato Sabaudo.

6 La rarità dei dizionari nei volgari d'Italia non toscani prima dell'Ottocento è nota: la Cornagliotti (REP: LXVIII n. 10) ne cita solo uno, bolognese, nel Seicento, e sei nel Settecento, rispettivamente spoletano, bresciano, veneziano e padovano, siciliano (“etimologico"!), napoletano, e roveretano e trentino (concluso però nell'Ottocento). Lessici “latino-volgari” (per parafrasare 1'etichetta di Gasca Queirazza 1972: v), anche se in misura assai più modesta di quelli latino-toscani, ne iniziano comunque ad apparire dal Cinquecento, tradizione su cui informa Ramello (2004: 61-63).

7 Le informazioni biografiche che abbiamo tanto sull'autore quanto sullo stampatore sono tutte in Gasca Queirazza (1972: VII-IX e IX-X); il Vopisco (1564) è peraltro citato nel 1859 dal Sant'Albino medesimo (X). 


\section{Peraltro}

il libro non dovette avere una larga diffusione, se si giudica dall'esiguo numero delle copie che oggi ne sono note. Oltre agli esemplari della Biblioteca Reale e della Biblioteca Civica di Torino ${ }^{8}$, della biblioteca privata della famiglia Cordero di Montezemolo, della biblioteca del cav. Vincenzo Armando, oggi dispersa, di quello del prof. Giuseppe Flechia, altri si trovano nella Biblioteca Universitaria di Bologna [Aul. V Caps. 177.30] e alla Bibliothèque Nationale di Parigi [X. 7494]?: in tutto sette. (Gasca Queirazza 1972: x-xi)

\subsection{Il Brovardi}

Saltando un paio di secoli, nella seconda metà del Settecento conosciamo in realtà due esperienze lessicografiche "piemontesi", una rimasta manoscritta ed una edita, appunto quella del Pipino.

Quello inedito è l'anepigrafico Dizionario piemontese, italiano, latino e francese o più brevemente Dissionari (REP: 1xii; CB 3123) del medico Gioachino Nicolao Brovardi ${ }^{10}$ consegnata a dieci volumi depositati all'Accademia delle scienze di Torino. L'opera è nota soprattutto per l'originalissima ${ }^{11}$ ortografia devisata dal Brovardi, su cui aveva attirato l'attenzione Âly-Belfàdel ${ }^{12}$ (1933: 361-66) e che si trova riassunta nel REP: lxiv-lxvi; ma come fonte era già stata usato dal Ponza (cfr. 1830-33: 1.VII) ed ora, estesamente, dal REP.

\subsection{Il Pipino}

Molto meno ricco ${ }^{13}$, ma diversamente insolito è il Vocabolario di Maurizio Pipino ${ }^{14}$ del 1783.

Un campione (p. 64b), tratto dalla sezione "domestica", dovrebbe bastare:

8 Questo forse era quando l'oggi era il 1972; ora che l'oggi è il 2018 a catalogo nella Reale e nella Civica vi sono solo le anastatiche allestite dal padre Gasca: visto che fu la Reale stessa a fornire l'esemplare di partenza per l'anastatica, è probabile che questo sia poi stato ritirato.

$9 \quad$ Le collocazioni sono date dal padre Gasca in nota.

10 Su cui si veda Cornagliotti (2002).

11 Per il tempo lo era senz'altro, anche se l'ispirazione prima, non so quanto confessata ma abbastanza evidente, sarà stata probabilmente la vecchia proposta ortografica del Trissino; comunque anche se quello è con buona verosimiglianza l'exemplum di partenza, certo il Brovardi si è spinto in là in una maniera inaudita: «era amico del precetto ortografico: ad ogni suono un segno, ed il suo metodo di scrittura è, a questo riguardo, perfetto», scrive Âly-Belfàdel (1933: 362), continuando «egli non badò ad alcuna legge ortografica in uso, calpestò tutto, dove trovò un vuoto, riempì, dove trovò un errore corresse», e concludendo che «il disegno è ardito; arditissimo poi ai tempi del Brovardi, in cui poco si parlava di modificazioni ortografiche, o se ne parlava inutilmente».

12 Interessante figura di medico (ancora una volta!) ed esperantista che, non pago di aver lasciato una Grammatica piemontese scritta finalmente nel linguaggio della glottologia d'oltralpe (anche se magari non con tutti i crismi; nelle parole, forse troppo dure, dei Clivio, CB 3089 «trattasi di lavoro nel complesso assai bene informato, ma che tradisce un'insufficiente preparazione scientifica»), poteva spaziare fino all'ungherese, di cui ha lasciato in italiano una grammatica relativamente pregevole; un personaggio, nel complesso, con i suoi limiti ma che meriterebbe qualche approfondimento monografico.

13 Sono solo 173 pagine più le 43 del Supplimento (177-220) e le 12 non numerate dell'Aggiunta (cfr. infra), per complessive circa 5.000 voci.

14 Medico alla corte di Vittorio Amedeo III, nato a Borgo San Dalmazzo nel 1739 e morto di peste nel 1788 durante un viaggio in India. Per il Pipino, oltre al classico Pacotto (1967: 390-96), ed indirettamente a Cornagliotti (1991), cfr. soprattutto ora Burdet (2004). 


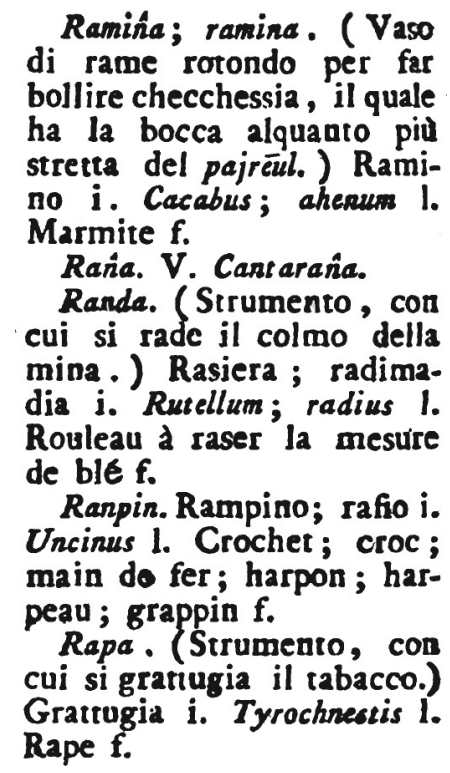

Anche qui le definizioni sono fornite oltre che in italiano, in latino ed in francese; l'italiano, però, è alquanto impreciso e si tratta perlopiù di mere perifrasi, neppure sempre adeguate. Che l'italiano sia, delle quattro lingue conosciute dal Pipino, la meno padroneggiata, e probabilmente la meno usata e l'ultima acquisita, è affatto probabile.

A parte l'ordine alfabetico talvolta approssimativo come peraltro normale nei dizionari dell'epoca, la cosa più notevole è che il lemmario non è continuo, ma diviso dispersivamente in diverse sezioni (più un «Errori, e mancanze occorse nella stampa», pp. 93-95): un «Vocabolario domestico» (pp. 1-92) con «l'Aggiunta al Vocabolario domestico» (12 pagine non numerate), una «Raccolta de' nomi Derivanti da Dignità, Gradi, Uffizi, Professioni, ed Arti» (97-134), una «Raccolta de’ verbi più famigliari» (135-173), ed infine un «Supplimento al vocabolario» (pp. 175-220) ${ }^{15}$.

Divisione curiosa che, al di là del rendere la consultazione difficoltosa, ha, almeno in parte, precise ragioni sociolinguistiche ${ }^{16}$; come dice la Cornagliotti (REP: LXXI) l'autore «è fortemente squilibrato a favore delle fasce selezionate, con evidente propensione verso le categorie alte della società in cui Pipino viveva e nella quale aspirava disperatamente di operare come medico e come uomo di cultura»; la consistenza, al di là già della sua mera presenza, della sezione dei Gradi la dice infatti lunga; ed a conferma della gravitazione dell'opera sulla corte di Torino, ed in definitiva del suo scopo, è che «la base linguistica [...] è essenzialmente il piemontese illustre di tipo torinese», (L. Ramello 2004: 31).

15 Per poco meno di 2.000 voci la sezione Domestica con la sua Aggiunta, circa 1.100 i Gradi, 600 i Verbi e 1.300 il Supplimento generico. Le cifre delle voci, qui e negli altri specimina menzionati, non sono mai direttamente fornite dai dizionari medesimi; sicché si danno solo delle semplici proiezioni statistiche, utilmente indicative ma per forza approssimate come tutte le statistiche.

16 Come nota L. Ramello (2004: 30), in ciò Pipino comunque mostra «una chiara sensibilità sulla questione della variazione diastratica». 


\section{Capello e Zalli}

Trent'anni dopo, nel primo ventennio dell'Ottocento, entriamo nella prima fase vera e propria di questa storia.

\subsection{Il Capello}

Nell'opera di Louis Capello, comte de Sanfranco ${ }^{17}$, secondo si autodesignava, la cosa più cospicua è il ruolo giocato del francese. Infatti, già avevamo rilevato l'implicita subalternità dell'italiano rispetto al latino ed al francese nel Pipino: qui tale subalternità si fa esplicita ${ }^{18}$. Ed oltre che la lingua, francese è anche la cultura di riferimento, enciclopedica ed erudita; di cui è buon testimone la produzione, abbondante ${ }^{19}$ anche se oggi spesso di difficile reperibilità, che spazia dalla cronologia universale (specie sacra), all'astronomia fino ad un dizionario di mitologia, Capello (1833), naturalmente in lingua francese, che dovette avere non poco successo, almeno in base alla sua (una volta tanto) ampia diffusione attuale ${ }^{20}$.

Ciò che immediatamente colpisce il lettore nel Dictionnaire portatif del Capello, uscito in due volumi nel '14 e frutto dell'epoca napoleonica, a parte la sua patente francofilia, è l'attenzione precipua all'aspetto terminografico, già fin dalla dicitura contenuta nel titolo ${ }^{21}$ e dalla struttura stessa dell'opera: tutto il secondo volume, intieramente francese, vi è infatti dedicato. Secondo avverte lo stesso Conte di San Franco nel Discours préliminaire (p. vii) anteposto al primo volume:

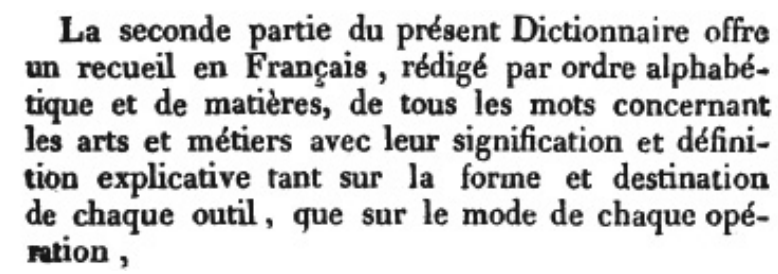

La componente terminografica, cui il REP, per forza di cose più interessato alla costruzione di un Piemontesischer Sprachschatz in senso diacronico ${ }^{22}$, è comprensi-

17 Sulla cui biografia informa una breve voce del Dizionario biografico Treccani: Ricci Massabò (1975), utilmente integrato dal REP: 1xxiii; cfr. anche L. Ramello (2004: 33-34).

18 Il rafforzamento del francese è certo effetto della mutata realtà politica: «con il ritorno dei francesi dopo Marengo e con il loro insediamento ormai saldo, la francesizzazione era destinata ad assumere i lineamenti di un vero e proprio programma di intervento, inevitabile dopo che l'11 settembre del 1802 veniva perfezionata l'annessione già votata nel 1799. Il Piemonte era parte della Francia, il francese entrava poco a poco nell'ordinamento scolastico, diventava lingua ufficiale dell'amministrazione e della burocrazia, lingua delle leggi, del diritto e quindi dei tribunali, incidendo così su un settore italianizzato fin dalla riforma di Emanuele Filiberto», secondo efficacemente riassumeva Marazzini (1984: 133-134), che poi proseguiva maggiormente a dettagliare (pp. 133-141).

19 Secondo riferisce la Ricci Massabò (1975); maggiori dettagli in Ronco (2012: 968).

20 È, ad esempio, tanto in Archive.org quanto in Google libri. «In esso l'autore offriva un'interpretazione scientifica della mitologia, analizzando e suddividendo i miti in "favole storiche, fisiche, allegoriche e morali" evidenziandone le differenze in base all'origine e alla provenienza geografica. All'opera fu riconosciuta dai contemporanei la dote della completezza e di un notevole approfondimento della materia. A riconoscimento di detti pregi 1'editore Pomba di Torino, nel 1837, iniziò con tale scritto la pubblicazione di una collana di dizionari» (Ricci Massabò 1975).

21 Vocabulaire français des termes usités dans les arts et métiers.

22 Cui cultismi, internazionalismi e prestiti recenti sono sostanzialmente un elemento di disturbo. 
bilmente abbastanza indifferente, è invece qui uno degli aspetti centrali. Questa naturalissima propensione non impedisce comunque al REP di formulare sul Capello un giudizio sostanzialmente equanime e ben meritato: «rispetto a Pipino, Capello si caratterizza per la più accurata ed equilibrata scelta dei lemmi; il suo Dictionnaire contiene quasi tutto ciò che appare nel Vocabolario pipiniano e i campi lessicali risultano più completi: aumenta il numero dei termini relativi ai mestieri e alle attività, ma il lemmario si estende anche a campi come la botanica, la zoologia, la chimica, l'alimentazione, ecc. II proposito iniziale si può dire raggiunto: sebbene fortemente vincolato alla terminologia francese, malgrado incertezze di chiarificazione e la difficoltà sovente manifesta di comprensione delle voci transalpine e una talora assai superficiale traduzione, Capello ha veramente fornito per primo ${ }^{23}$ un Dictionnaire al piemontese» (REP: 1xxv).

«Nel primo tomo Capello inserisce poi quei proverbi piemontesi che è riuscito a rendere adeguatamente in francese conservandone lo spirito» (L. Ramello 2004: 35); l'osservazione è preziosa, perché l'attenzione paremiologica, oltre che essere parzialmente ereditata anche dal Sant'Albino, diventerà una delle marche di fabbrica del Viriglio (cfr. infra).

\subsection{Lo Zalli}

Il dizionario dello Zalli ${ }^{24}$ seguì a ruota l'anno dopo, nell'anno di grazia 1815 , presso una stamperia carmagnolese di grande tradizione, quella di Pietro Barbiè, che della seconda edizione, postuma, si farà poi carico da solo nel '30.

In tre volumi, di grande impianto ${ }^{25}$, segna tutto sommato un ritorno al Pipino (nella struttura, certo non nelle dimensioni ${ }^{26}$ ), non solo nel trilinguismo dei referenti, che viene anzi molto sistematizzato (alla reintroduzione del latino si accompagna quella di un italiano meno deficitario), anziché riprendere il solo francese del Capello (anche perché dopo il Congresso di Vienna è mutato il clima politico), di cui si abbandona anche la prospettiva terminologica, ma soprattutto nei criteri sociolinguistici, che così lo Zalli esplicita nella Prefasion al primo volume (p. 2):

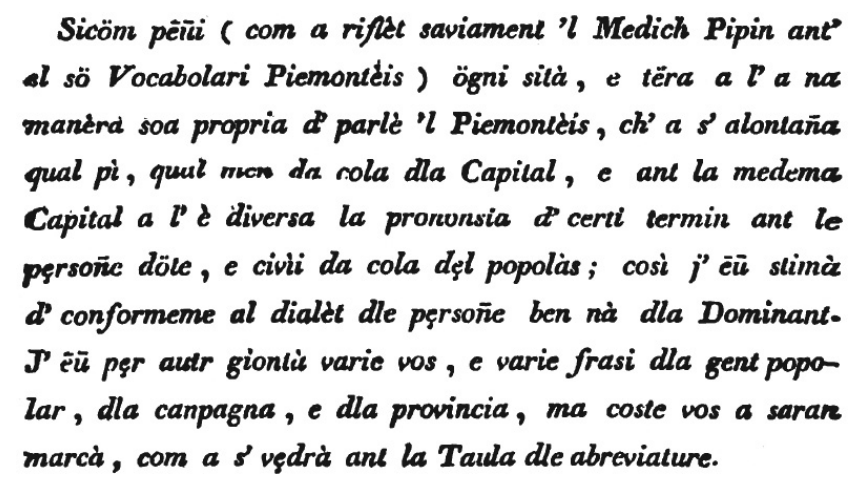

23 Già solo dal punto di vista quantitativo: il tomo piemontese novera c. 25.000 voci a fronte delle 5.000 del Pipino.

24 Su Casimiro Zalli, prelato di Chieri, ben poco si sa oltre alle date 1754-1817: cfr. REP: lxxv, n. 49.

25 Inoltre, è anche posposta una cospicua Gionta (pp. 245-603), che lo Zalli medesimo dichiara attinta al Capello.

26 In tutto vi sono circa 25.000 voci, compresa la Gionta: come nel Capello, quindi, ma più ampie. 
Diacoricamente, come pure si desume, netta è la scelta (come già nel Pipino, ma pure nel Capello) per la varietà della capitale, decisione che si perpetuerà tutto sommato fino ad oggi, per cui "piemontese" non vale altro che "torinese (illustre)".

Con la seconda edizione, non più a cura dell'autore, ed in "soli" due volumi ${ }^{27}$, ci si inoltra già nell'era seguente: la pubblicazione dei suoi fascicoli, infatti, si intreccia con quelli della "prima" (cfr. paulo infra) ed. del Ponza, con cui il Barbiè intrattenne una polemica senza esclusione di colpi che è stata ben ricostruita da Ronco (2012). Che poi anche il Ponza si sia attirato gli strali di altri critici, è forse giustizia divina: si veda almeno G. A. Ramello (1831), ma altri ${ }^{28}$ ne menziona la CB: 192.

\section{Il Ponza}

Entrando, con gli anni Trenta dell'Ottocento, a questo punto nella galassia Ponza la bussola si sposta definitivamente sull'italiano. Dico galassia perché davvero molte sono le edizioni del Ponza, e difficile è orientarvisi.

Il personaggio stesso, l'abate Michele Ponza (1772-1846), è poliedrico, anche se caratteristiche individuanti sono il suo interesse didattico ed il suo purismo oltranzista. Quanto al primo aspetto ${ }^{29}$, è senz'altro un notevole innovatore ${ }^{30}$ : amico di Rosmini e fautore di Kant ${ }^{31}$, pensava che per insegnare l'italiano bisognasse partire dalla madrelingua degli allievi, cioè dal piemontese; donde la sua attività lessicografica. Quanto al secondo, il Ponza si inserisce certo in una ricca tradizione di purismo piemontese, di cui le maggiori eccellenze sono l'ảbate di Valperga Caluso e Vittorio Alfieri $^{32}$, ma ne trae le conseguenze più intransigenti. Fondò e diresse prima l'Annotatore degli errori di lingua, che uscì a cavallo degli anni Trenta per sole tre serie, e poi per più anni (dieci numeri), dal 1835 al 1839, l'Annotatore piemontese, entrambe "riviste di letteratura italiana" puristicamente orientate. Ovvio che tale impostazione lo avesse portato in aperta polemica coi dizionari precedenti, rei di lesa italianità, quando non di peggio.

Limitandoci ora alle sole opere propriamente lessicografiche ${ }^{33}$, nel corso della sua vita uscirono ben sette ${ }^{34}$ specimina: cifre da capogiro; e non è un caso che la non troppo accurata bibliografia dei Clivio ad un certo punto perda la bussola...

27 Il dato non tragga in inganno, perché se le voci complessive scendono a circa 20.000, pure vi sono due appendici (risp. di 115 e 36 pp.), sulla cui costituzione vedi Ronco (2012), con grossomodo 4.500 aggiunte e correzioni. Inoltre, mediamente, si assiste ad un ampliamento delle voci già notato ed illustrato da L. Ramello (2004: 40).

28 Soprattutto un agguerrito «mastro Leonardo Ciabattino», cui sarà da aggiungere (in questo florilegio di sedicenti artigiani) il «mastro Simone Barbiere» critico dell' Annotatore degli errori di lingua.

29 Insegnare l'italiano ai piemontesi era d'altra parte il suo principale mestiere: quello di Maestro di grammatica e di Prefetto delle scuole di Porta Nuova.

30 La sua "progressività", che lo ha spesso fatto scontrare con le dirigenze, è ben documentata nel REP: lxxvii.

31 Aspetti indubbiamente degni di approfondimento, che qui dovremo abbandonare per limiti di spazio.

32 È in questo clima che va inserita la pubblicazione (facilmente reperibile anche su Google libri: https://books. google.it/books?id=a609LIDsk9IC) nel 1827 da parte di Luigi Cibrario di quei Modi Toscani raccolti dall'A1fieri in un "quadernuccio" tra il 1778 ed il 1802: in sé testimonianza di un'era precedente, di un uomo che l'italiano se lo dovette imparare almeno come quarta lingua sudando in quel modo "eroico" (e molto alfieriano) che lui stesso racconta nella Vita, ma che Cibrario funzionalizza al purismo a lui contemporaneo.

33 È, d'altra parte, per la sua attività di lessicografo che è meglio noto nella storia della linguistica, anche se in realtà è un lessicografo del piemontese malgré lui: centrale nel suo "programma" è l'italiano, da cui discende tutto il resto. Sugli altri aspetti cfr. ora Barbera (2018 in stampa).

34 Contando per buona l'edizione del 1847, propriamente postuma, ma ancora verosimilmente curata dall'autore. 
Il taglio superiore è relativamente chiaro: Ponza pare che morisse alla fine del 1846, ma la prefazione ( «Il compilatore sacerdote Michele Ponza ai benevoli suoi lettori», pp. iii-xi) all'edizione del '47 (riprodotta dal '46) è ancora sua, e l'edizio$\mathrm{ne}^{35}$, praticamente postuma, è l'ultima ad essere d'autore. Le successive, dal 1859 in poi, sono solo operazioni editoriali che riproducono ecletticamente il testo del 1847 , potato di non poche voci, ma addizionato di molte altre recuperate diversamente (sicché l'edizione del '59 si presenta apparentemente più completa della precedente in quanto più "lunga", anche se non ha la parte italiano - piemontese ma solo la Teorica, che riproduce dall'edizione del '47); in particolare la cosiddetta "sesta" del 1860 è una perfetta descripta della "quinta" e così via. Se si tiene come punto fermo la cosiddetta "quarta" del 1847, il rimanente è più confuso.

Dalla prefazione all'edizione del '47 (p. iv, che riproduce le medesime parole di quella del '43, p. i), si dovrebbe considerare "prima" l'edizione estesa in tre volumi principiata nel 1830 e conclusa nel $1833^{36}$. Edizione, però, che, come detto in quella prefazione, era stata preceduta da altre due prove che è difficile rintracciare. Quella del $1826^{37}$ CB 3129, menzionata anche dal REP: lxxviii, n. 70, dai Clivio e da Marazzini (2012: 98-99), costituiva il secondo volume di un Inviamento $^{38}$ al comporre nella lingua italiana, mentre di quella del '27 CB 3130 e della sua natura "scolastica" ${ }^{19}$ non ho altre notizie che quelle fornite dallo stesso Ponza, che nella «Premessa» alla sua edizione del '30 (pp. vi-vii) dice chiaramente che si tratta di una mera seconda edizione di quella del ' 26 :

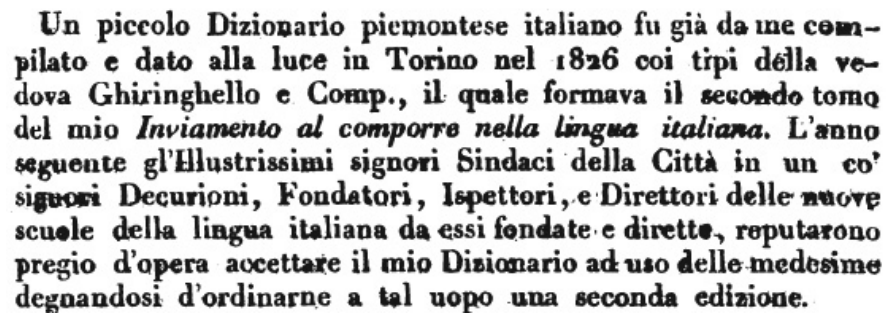

A tutti gli effetti, quindi, il magnum opus nasce con la forma che assume nel '30, e che l'autore medesimo considera la "prima edizione" ${ }^{40}$, ed è sostanzialmente sganciata dall'altra serie (cfr. infra).

Porre correttamente in serie l'edizione successiva alla "prima" del '30-33, poi, non parrebbe troppo difficile, e comunque vi sopperirebbe lo stesso Ponza nella prefazione del '47 (pp. iv-v),

35 Che riproduce in testa al dizionario quella «Teorica dei nomi e dei verbi italiani» apparsa per la prima volta in questa veste nell' «Appendice» all'ed. del 1844, ma di cui una prima versione apriva già l'ed. del '30; cui aggiunge una «Teorica di gramatica piemontese - italiana» che riproduce la prima parte del suo Donato Piemontese del 1938.

36 Almeno così recitano i frontespizi; l'uscita dei fascicoli si sarà però forse protratta ancora l'anno successivo, visto che la prefazione pare scritta nel 1834.

37 Giovanni Ronco me ne ha segnalato (c.p.) la presenza di una copia alla Nazionale di Torino; un'altra copia è alla Biblioteca storica della Provincia di Torino. Assolutamente introvabile è invece l'edizione del '27 per cui sovvengono solo le menzioni dello stesso Ponza, CB 3130 e Gasca Queirazza (1967).

38 Il secondo volume era però intitolato Istradamento, ecc.

39 Comune anche alla precedente, ed alla "serie" tutta: nacque infatti nel '26 come secondo volume di quel Inviamento al comporre nella lingua italiana di cui s'è già detto.

40 Le precedenti due potrebbero essere intese come le "zero" e "doppio zero", alla stregua delle sinfonie di Bruckner. 
Nel 1843, trovandosi esausta anche l'edizione di questo, pensai d'aceingermi a darne una seconda con molti miglioramenti e non poche rettificazioni ed aggiunte, facendo ad esso succedere il Vocabolario Italiano-Piemontese, desiderato da moltissimi,

sennonché prima, nel 1834, esce un Dizionarietto che sul frontespizio si qualifica come "terza edizione corretta ed ampliata": ma terza di cosa? La risposta, come consueto, è data dal Ponza medesimo nella prefazione (p. 4):

6. Saranno comprese in questo $\mathrm{Di}$ zionarietto le voci che mancano al $\mathrm{Di}$ aionario piccolo stampatosi dalla tipografia GhiringheHo nel 1826 , e faciente il If tomo dell' Inviamento al comporre nella lingua italiana, il quale ebbe separatamente uaa seconda edizione nel 1827 per mezzo dèi tipi medesimi, ai cui moltiplici difetti d'omissione e di non troppo giuste definizioni ehe sfuggirono in quelle due edizioni vuole ora l'autore di esso riparare con questa rerza, dichiarando apocrifa, e difettosa ogni altra successiva ristampa delle due succitate edizioni.

Cioè si avrebbe una serie di tre edizioni ${ }^{41}$ di un'opera, che per semplificare potremmo chiamare Dizionarietto: nata nel '26 e resuscitata nel '34, fu concepita per pure funzioni didattiche istituzionali; ed una serie di un'altra opera, che nasce nel '30-33, affatto più generale (ed in cui le istanze didattiche stanno dietro, ma non sono così esplicite), che potremmo chiamare Vocabolario ${ }^{42}$.

Non facile, infine, è pure calettare cosa sia intervenuto tra la "seconda" e la "quarta". Il primo spunto viene, di nuovo, da un passo (p. v) della solita prefazione ponziana all'edizione del '47:

\section{[...]; indi una terza nello}

scorso anno 1846, riveduta ed ampliata di circa 2000 voci. Il rapido smercio di codeste edizioni ne prova abbastanza il generale aggradimento, ed una quarta vede di bel nuovo la luce.

Ora, tanto 1'edizione del '44 quanto quella del '46 sono dichiarate "terza" anche se sono diverse; in particolare la "terza" del '46 risulta identica alla "quarta” del '47,

41 Come aveva già visto il padre Gasca: «il primo [tempo] dà come frutto un modesto dizionario, nato a servizio della scuola, come necessario complemento allo Inviamento al comporre in lingua italiana, pubblicato nel 1826 e ristampato nel 1828 e 1834 con ampliamenti, tra le 112 e 166 pagine» (Gasca Queirazza 1967: iii; devo la segnalazione a Giovanni Ronco).

42 Continuando ad usare la convenzione "bruckneriana" potremmo arrivare ad una "triplo zero", usando a questo punto, per comodità, l'ordine inverso, per cui se la "Nullte" è la sinfonia più vicina alla prima, qui è l'edizione più lontana da quella del '30-33. 
e comunque tutte le "terze" sono oggi assai rare, probabilmente per le ragioni che si indovinano dietro la dichiarazione di Giuseppe Lobetti-Bodone, il tipografo che aveva acquisito i diritti da Carlo Schiepatti (l'editore del '47), nella prefazione alla sua "quinta" edizione del '59 (p. 2):

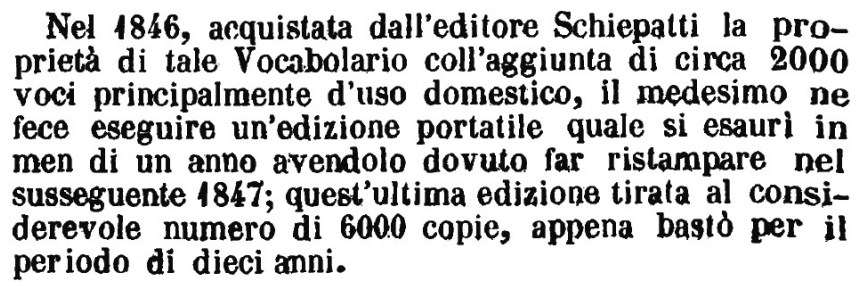

E che sono allora queste edizioni?

A cambiare è anche lo stampatore, e questi "cambi di cavallo" durante la corsa e la fenomenologia sopra accennata fanno pensare a qualche torbido editoriale (più $\mathrm{o}$ meno sottaciuto) e che la "seconda terza" sia solo una prova d'orchestra per la "quarta", ad essa di fatto uguale.

Comunque, la "prima terza" è solo una forma di transizione ${ }^{43}$ tra la "seconda" 44 e la "quarta", che ha avuto una diffusione tumultuosa tra il 1844 ed il $1846^{45}$, ma che si poteva considerare conclusa (questo il senso dell'affermazione del Ponza nella prefazione del '47) tanto dal punto di vista redazionale che da quello editoriale. In altre parole: la "terza edizione" ha quindi due forme, quella del ' $44 \mathrm{e}$ quella del ' 46.

La seriazione complessiva ${ }^{46}$ che così si otterrebbe è: 1826 " 0 "; 1827 " 00 "; 183033 “1”; 1834 “000”: 1843 “2”; 1844 “3a”; 1846 “3b”; 1847 “4”; 1859 “5”; 1860 “6”; ecc: si tratta, come s'è detto, di due serie distinte: una minore, il Dizionarietto, e l'altra maggiore, il Vocabolario. La prima conosce tre edizioni, che vanno dal '26 al '34 (circa 8.000 voci) e l'altra quattro, dal '31 (in tre tomi di circa $20.000^{47}$ voci) al '47 (tomo unico di circa 13.000 voci) senza contare le molte edizioni postume ${ }^{48}$ : queste, come si diceva, sono solo operazioni editoriali che riproducono ecletticamente il testo del 1847.

Che l'italiano sia comunque l'interesse centrale anche nella serie maggiore, basti a confermarlo un sommario esame del campione seguente, tratto dalla quarta edizione del Vocabolario, Ponza (1847: 300a)

43 Ne è prova l'ampiezza assunta dall'appendice e l'indicazione curiosa, e quasi mendace, di "due volumi".

44 È semmai da questa edizione che si inizia ad abbozzare, via via accrescendosi, una forma nuova che abbandona quella in tre tomi del '30-'33; qui si sono ritenuti più forti gli elementi di continuità considerando una sola opera, articolata in una serie di edizioni successive; altri, come Gasca Queirazza (1967), ha preferito costituire due tempi distinti, di cui uno si arresta con le «Appendici» (che abbiamo un poco trascurato) alla versione del '30-'33.

45 Probabilmente nel '46 è stata anche stampata in una tiratura insufficiente (questo si legge tra le righe del Lobetti-Bodoni riportate in Tav. 9) da un editore che non si trovava troppo sicuro del suo locum standi.

46 Non tengo conto dell'appendice al Donato CB 3265 (pp. 137-140), chiaramente surrettizia, e di quella all' Antologia CB 3139=3266 (pp. 117-132), un poco più ampia ma dello stesso tenore: sono diverse perché sono diverse le antologie cui forniscono un glossario, ma le funzioni sono le medesime.

47 Quantitativamente poco al di sotto delle 25.000 del Capello e dello Zalli prima edizione.

48 A mia conoscenza, comunque, non si va oltre la nona. 


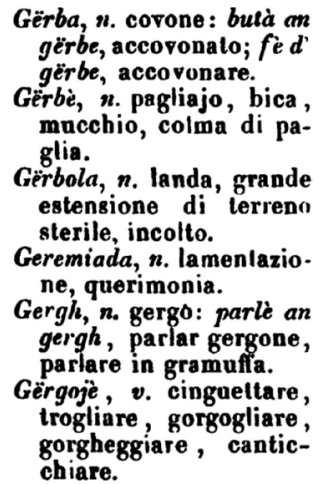

Si noterà come l'attenzione sia evidentemente rivolta più all'italiano che al piemontese: i rapporti derivazionali, ad esempio, tra gërba, gërbé e gërbola non sono esplicitati, mentre sono espanse le sinonimie delle rese italiane, che nel caso di gërgojé giungono a cinque ${ }^{49}$. Tra queste, peraltro, si annidano sicuri toscanismi di marca cruscante, come il vistoso parlare in gramuffa ${ }^{50}$, ancora assente nel ' $32^{51}$.

\section{Il Sant'Albino}

Prima dell'uscita nel 1859 del Sant'Albino, che è tuttora considerato il momento culminante della tradizione lessicografica piemontese, oltre che una ripresa (pressoché coeva all'uscita del magnum opus) postuma del Ponza (cui ne seguirono molte altre), si registra solo il piccolissimo, ma pregevole, Saggio di vocabolario pedemontano CB 3144 contenuto tra le pp. 557 e 577 del Saggio gallo-italico del Biondelli. Cioè, in definitiva, v'è comunque ben poca cosa: è Sant'Albino ad improntare di sé gli anni Sessanta ed i successivi (e praticamente, attraverso le molte anastatiche, la sua autorevolezza perdura fino ad oggi).

La biografia del personaggio, dopo lo studio di Bianco (2003), efficacemente riassunto anche dal REP: 1xxviii-lxxx, è complessivamente ben nota e non mette conto di raccontarla nuovamente; invece concentriamoci sul vocabolario.

La cui eccellenza è indubbia, non fosse che quantitativamente: secondo i calcoli del Bianco (riportati anche dal REP: 1xxx), infatti, «comprende 3000 voci in più rispetto a Zalli» ${ }^{52}$, stima cui l'hanno forse condotto le dichiarazioni del Sant'Albino medesimo nella sua «Prefazione» ${ }^{53}$; opere di pari impianto sono forse solo il Cherubini per il milanese ed il Boerio per il veneziano. Anche le cronologie sono compa-

49 Che nella prima versione del Vocabolario erano addirittura dieci: cfr. Ponza (1830-33: II.96b).

50 «GRAMUFFA. Favellare in gramuffa, si dice in ischerzo per Favellare in gramatica, quasi in modo da non

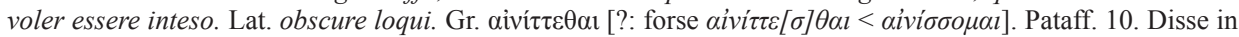
gramuffa, mostrando malpiglio» (Crusca veneziana: II.449a = Crusca veronese: III.306c).

51 Il passaggio dalla versione in tre volumi a quella in volume unico si è infatti accompagnata ad un asciugamento del lemmario, ad un meno vistoso ma assai importante lavoro di perfezionamento formale (vedi la notazione di $<\ddot{e}>$ laddove la pristina edizione aveva semplicemente $<\mathrm{e}>$ ), ma soprattutto ad una robusta risciacquatura cruscante.

52 Il calcolo è probabilmente per difetto: secondo la mia valutazione le voci dovrebbero essere almeno 30.000 .

53 Don Vittorio parla di averne accresciuto «il novero di ben due o tre mila sopra quello del Zalli, che era finora il più ricco di ogni altro», Sant'Albino (1859: xii). 
rabili ma non identiche: il Cherubini è precedente di poco (è concluso nel '56, ma la sua composizione si intreccia con quella del Sant'Albino ${ }^{54}$ ), il Boerio, invece è precedente di molto (venne terminato nel '29), ma ve ne fu una seconda edizione (procurata dal Cecchini), postuma ed aggiornata anche con «l'Indice italiano - veneziano», proprio nel ' $56^{55}$; uno degli intenti di Sant'Albino è certo quello di inserire il piemontese nel generale concerto «delle contemporanee grandi opere linguistiche che in tutta Italia qua e là si pubblicavano» (REP: lxxx, che ne fornisce un buon elenco).

Ma oltre che nell'ampiezza del lemmario è soprattutto nella campitura delle voci (in cui è specialmente la componente fraseologica ${ }^{56}$ ed in particolare sinonimica ad essere irrobustita) che si vede la grandezza del Sant'Albino ${ }^{57}$, anche se il solco è pur sempre quello tracciato dal Ponza: «la parlata materna ${ }^{58}$ in funzione della comprensione e dell'acquisizione dell'italiano» (REP: 1xxxii). Le fonti, su cui ha gettato luce in particolare il REP: 1xxx-lxxxi, al di là dei dizionari piemontesi precedenti ${ }^{59}$, sono infatti soprattutto italiane, anche se notevoli: oltre ovviamente alle varie Crusche (ed in ispecie alla quarta), a far banco è l'innovativo Tramater, cui sono associati i Sinonimi del Tommaseo, il Villanuova ed il Carena ${ }^{60}$; tutte fonti eccellenti, spesso neppure ovvie, ma che confermano quel generale orientamento verso l'italiano di cui si diceva.

Giustamente nel REP: 1xxxi-lxxxii si ribadisce che «sulla metodologia adottata dal Di Sant'Albino per la sua opera va tuttavia effettuata una ricerca a parte che consideri la sua creazione un punto di partenza unico per [la] lessicologia di nuova

54 Di cui è noto il tentativo nel '47 di pubblicare in proprio le prime dispense del Vocabolario, presto interrotte dalla guerra di indipendenza ma soprattutto dalla difficoltà di fronteggiare i costi della stampa, cfr. Bianco (2003: 458), fino a completare l'opera e darla alla stampa presso la UTET dieci anni dopo: «la prefazione degli editori è del $1^{\circ}$ gennaio 1859, ma l'edizione fu completata solo l'anno successivo» (Bianco 2003: 458).

55 Ed a questa ne seguì una terza, definitiva, nel 1867.

56 Già punto di forza del Ponza, diventerà non a caso, ma finalmente per il piemontese e non per l'italiano, il cavallo di battaglia del Viriglio, cfr. infra.

57 Evidente anche in molti dettagli, come quello dell'ortografia: «finalmente oltre varie altre innovazioni, si è introdotto un metodo ortografico affatto nuovo, atto a facilitare la pronuncia e la retta interpretazione delle voci del nostro dialetto, come si può vedere alla Tavola delle avvertenze» (Sant'Albino 1859: xii). In questo campo, in cui Brovardi aveva molto contribuito (restando, non a caso, manoscritto), ed in cui il Barbiè aveva gettato la spugna («Era nostro pensiero il pubblicare questa seconda Edizione con nuova ortografia, sia perchè gentilmente ci vennero favorite le diverse accentature da Persona che molto stimiamo; sia perchè, a dir vero, a Noi stessi sembravano queste più accomodate; ma quand'anche pel zelo e desiderio di far cosa grata al Pubblico avessimo già specialmente fatte gettare a nostro carico tutte le lettere opportune, prima però di giungere a simil passo, avendo consultato varie erudite Persone delle diverse Provincie, avemmo in riscontro da quelle, che la stessa lettera segnata da alcuni coll'é acuto era da altri corretta coll'è grave; e che l'o voluto stretto da certuni veniva scritto ö largo da cert'altri; e noi frattanto passavamo sfaccendati le settimane menando per le lunghe la Stampa, pel puro desiderio di far cosa utile e cara al Pubblico, ma quindi stanchi dal continuo variar di massime, dotte Persone facendoci opportunamente osservare essere in primo luogo difficile l'adattare un'accentatura, che corrisponda al vario articolar delle sillabe, essendo quella dissimile nelle diverse Città e Terre; e secondariamente non dover essere nostro scopo il creare nuova accentatura, ma bensì di porgere i significamenti italiani, latini e francesi a ciascun vocabolo o frase piemontese, e non già d'insegnare ai Piemontesi la maniera di scrivere nel nostro Dialetto, onde, dietro a questi riflessi, riputammo più saggio consiglio l'attenerci quasi strettamente all'accentatura già praticata dallo stesso Zalli, come la più conosciuta, lasciando a dotte penne la gloria del nuovo punteggiare, e segnare le sillabe di doppia pronuncia», Zalli 1830: 1.IV), era stato ancora una volta il Ponza a distinguersi, molto migliorando dalla prima alla quarta edizione del suo Vocabolario.

58 L'amore per la quale («il dialetto piemontese, quantunque non difetti di grazia e di brio, sia assai ricco di vocaboli, ed abbia il pregio di una straordinaria concisione e vigoria, è forse più che ogni altro sconosciuto al resto d'Italia», Sant'Albino 1859: ix) è peraltro indubbio e viene professato più volte nella «Prefazione».

59 Ma non il manoscritto del Brovardi, che sembra non conoscere: cfr. Cornagliotti (2002: 341).

60 Altro grande piemontese, di Carmagnola, che pubblicava a Torino. 
specie che nasce in Piemonte»; ricerca che qui naturalmente non si può varare, contentandoci di un paio di esempi, per meglio illustrare la sua operazione.

Il primo (da p. 626a) getta luce soprattutto sulla ricchezza sinonimica, ma evidenzia anche come il moltiplicarsi dei sinonimi riguardi solo l'italiano:

Ghice. Bugigatto, bugigattolo, piccol buco, bucherattolo, pertugio; ed anche piccolo stanzino, ripostiglio, stambugio o stambugello.

Ghicet o Usset. Sportello. Piccolo uscetto in alcune porte grandi, od in quella delle botteghe, a guisa di finestra.

- Talora dicesi per Ghicc. V.

Ghidon. V. Guidon.

Ghiga o Ceca. Buffetto. Colpo di dito che scocchi di sotto un altro dito.

Ghigh. V. Gabriolè.

Ghign. Ghigno, sghigno, sogghigno. L'atto del ghignare. Tacito sorriso quasi per beffa o disprez.zo.

Ghigna. Luchera, ceffo, mostaccio. Labbia, aria del viso, aspetto. Un certo modo di guardare, altr. piglio.

- A l a una certa ghigna ch'am pias poch. Egli ha una certa luchera o lucheria, un certo aspetto che non mi va a sangue; e più intens. egli ha un ceffo di tristo, un viso, una faccia torva, cioè un aspetto che ributta, che incute terrore.

Il secondo (da p. 947ab) illustra come comunque nelle parti più grammaticali l'aspetto sintattico sia ignorato a fronte di quelli fraseologici e sinonimici:

Q:iand. av. Quando, allora che, in quel tempo che.

- Quando, in qual tempo.

$"$ - Qualora, ogni volta che.

Quand ben. Quando bene, ancorchè, benchè, quantunque, comechè, sebbene.

- s'sia. Quando che sia, ad ogni tempo, qualunque volta, in qualunque tempo si voglia, ognora, semprechè..... Ogni volta che.....

- Anche quand. V. prima Quand ben.

- D'quand in quand. Di quando in quando, a quando a quando, di tempo in tempo, a tempo a tempo, alle volte, alcuna volta.

Si confronti anche solo la voce quando con quella del Costa / Cardinali (1819-26: 5.565ab) o del "conservatore" Manuzzi (1833-40: II.1.694b-695a). Ed analoghe risultanze si avrebbero da altri campioni.

La linea "italiana" sancita dal Ponza emerge comunque netta: la più grande opera lessicografica piemontese, paradossalmente, ne sancisce issofatto la morte. Questo, se è già evidente nella costruzione stessa delle voci, è anche chiaramente espresso nella «Prefazione», (Sant'Albino 1859: 10-11), dove il riferimento a Giordani è particolarmente chiarificatore della natura risorgimentale (e "moderata" ma non per questo meno letale) della scelta di campo per l'italiano come lingua nazionale e come strumento per "mantenere e diffondere la civiltà" (per usare espressioni giordaniane), promuovendone le classi inferiori. 
A parte sottolineare le ragioni sociolinguistiche di certi atteggiamenti, cercherei però almeno di evidenziare alcune trame storiche che, accanto alla qualità intrinseca dell'opera, ne hanno decretato l'importanza, prima di tutte la scelta ${ }^{61}$ dell'editore: la da poco nata (nel 1854) UTET, l'ultimo frutto delle fatiche di Giuseppe Pomba. Non significava solo entrare nelle mani di uno degli editori più vulcanici della storia dell'editoria italiana, e quindi far parte della scuderia di uno dei più dinamici grup$\mathrm{pi}^{62}$ editoriali d'Italia, ma anche comparire come parte integrante di quella linea (le ragioni risorgimentali che ritornano!) che partendo dall'Enciclopedia popolare giungerà fino al Tommaseo ${ }^{63}$; anzi, nell'avvertenza editoriale in testa al volume è detto esplicitamente: «non mancano certamente in Italia Vocabolarii dei varii dialetti, ma i più di essi sono insufficienti e incompiuti. In Piemonte ne abbiamo avuti parecchi; [...] ma oltrechè sono tutti, qual più qual meno, incompleti; eccetto l'ultimo, divennero rarissimi, e quello del Zalli quasi irreperibile. Queste considerazioni indussero la nostra Società ad occuparsi della pubblicazione di un buon Dizionario Piemontese-Italiano, nell'intendimento di fare cosa veramente utile, e quindi anche per facilitare tra noi lo smercio del più volte menzionato nostro Gran Dizionario della Lingua Italiana ${ }^{64} \gg$ (Sant'Albino 1859: vi).

\section{Pasquali e Gavuzzi}

Con l'uscita del Sant'Albino il canone si fissa, e le altre voci non possono che spegnersi (come gradualmente avvenne al Vocabolario del Ponza, di cui si conoscono stanche repliche fino agli anni Settanta), ignorarlo (come fece il Pasquali), o cercare diverse vie (come tentò il Gavuzzi).

\subsection{Il Pasquali}

Piccolo (è in $16^{\circ}$ ) nonostante l'elevato numero di pagine ${ }^{65}$, è il prodotto più depresso di questa fase. Per darne un'idea basti concentrarsi su un aspetto che abbiamo trascurato: le etimologie. Pasquali ${ }^{66}$ promette esplicitamente (cosa abbastanza insolita) anche etimologie, ma la realtà è che queste o sono assenti o sono (per essere gentili)

${ }_{61}$ O meglio, se si presta credito alla prefazione editoriale («E cotal suo divisamento [la pubblicazione di un buon Dizionario Piemontese-Italiano] ebbe ventura di poter mandare ad effetto, poichè [la nostra Società] trovò un lavoro di questo genere finito e in pronto per opera del cav. Vittorio di Sant'Albino, il quale fin dal $1847 \mathrm{ne}$ aveva intrapresa per proprio conto la pubblicazione», Sant'Albino 1859: vi) fu Sant'Albino ad essere stato scelto da quell'editore.

${ }_{62}$ Gruppo con cui la sintonia dovette essere certo precedente, se ad esempio quello Zecchini, con cui il Sant'Albino condivise la passione per i sinonimi, di Giuseppe Pomba era genero.

63 Per l'UTET ed il Tommaseo cfr. Fanfani (2016) ed alcuni dei contributi in Beccaria / Soletti (2005).

${ }_{64}$ Questo riferimento nel 1859 può sembrare un poco de iure condendo, se si pensa che i volumi sono usciti tra il 1861 e il 1874; ma ci si dimentica che l'impresa era partita già da gran tempo: i primi contatti tra Tommaseo e Pomba risalgono al 1835, anche se è "solo" nel '53 che il progetto venne chiaramente definito; del '56 è la firma del contratto e del '57 l'inizio della stesura vera e propria. Il richiamo nel '59, quindi, non giunge affatto "fuori tempo": all'inizio dei lavori a quella che doveva diventare l'impresa più importante del Pomba, il pensiero ad essa doveva essere certo dei più pressanti.

65 Ha comunque almeno 10.000 voci.

${ }_{66}$ Di Giovanni Battista Pasquali nulla so, se non che si autoqualifica come "professore". 
deficitarie. Bastino due casuali ${ }^{67}$, ma tipici (e certo non dei peggiori) assaggi, uno dalla p. 11a dell'ed. 1870 ed uno da p. 451b dell'ed. del 1869:

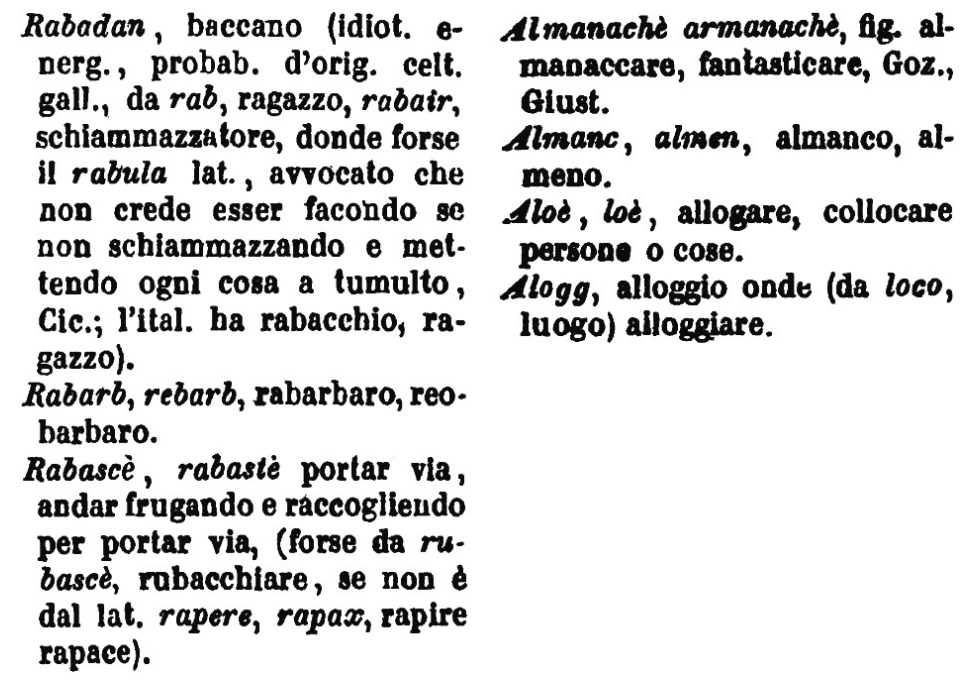

Si noterà come a volte la reticenza e l'imprecisione cedano alla fantasia, e come la celtomania ottocentesca evidentemente la spadroneggi. E forse questa è la ragione della sua sostanziale assenza nel $\mathrm{REP}^{68}$, cosa che a tutta prima stupisce un poco: si tratterà più di pudore che di trascuratezza...

\subsection{Gavuzzi}

Fisicamente più "piccoli" del Sant'Albino, ma pur sempre più "grandi" del Pasquali (entrambi i dizionari sono in $8^{\circ}$ intorno alle 700 pagine), i Gavuzzi ${ }^{69}$ hanno certo ben altra dignità di quest'ultimo.

Tanto per iniziare da quello più "normale", cioè il piemontese - italiano Gavuzzi (1891), esso novera circa 30.000 voci $^{70}$ e non è completamente dipendente dal Sant'Albino, anzi tutt'altro. Prendiamo un campione a caso (parte della sequenza rap-, Gavuzzi (1891: 510a)

Per cui cfr. invece il REP: 1157, 1165 e 16 sub vv. rabarb, rabasté, ramadan e aloé.

68 Pasquali è citato dal REP solo tangenzialmente; ben due pagine gli dedica invece L. Ramello (2004: 51-52).

69 Anche sul Gavuzzi purtroppo non mi soccorrono molte informazioni biografiche, se non che era un ingegnere di Caramagna e che fu impiegato dal Ministero delle Finanze (entrambe notizie fornite da lui medesimo). Assente nel Dizionario biografico degli italiani, non è comunque da confondere con lo Stefano Giuseppe Antonio notaio ed autore del famoso Adramiteno, come spesso è capitato: entrambi avevano lo stesso cognome ed erano piemontesi, ma floruerunt ad un secolo di distanza ed uno si chiamava Giuseppe e l'altro Stefano, e solo di secondo nome Giuseppe. Sullo Stefano Giuseppe Antonio mi sovviene, peraltro, solo una scarna voce nella Treccani online: «Gavuzzi 〈-zzi〉, Stefano Giuseppe Antonio. - Giurista ed epigrammista (Torino 1709 circa - Vinovo 1782). Fu senatore a Torino. Ebbe fama per le strofette dei pronostici nel Palmaverde, per le Favole d'Esofago da Cetego, parodia delle favole settecentesche, e, soprattutto, per l'Adramiteno (post., 1790), parodia degl'insulsi libretti per melodramma del tempo. Collaborò, anonimo, alla Pratica legale (1772) di P. G. Galli della Loggia».

70 Cioè quantitativamente il numero delle voci è praticamente pari a quello del Sant'Albino; va da sé che la dimensione dei lemmi è mediamente assai minore. 


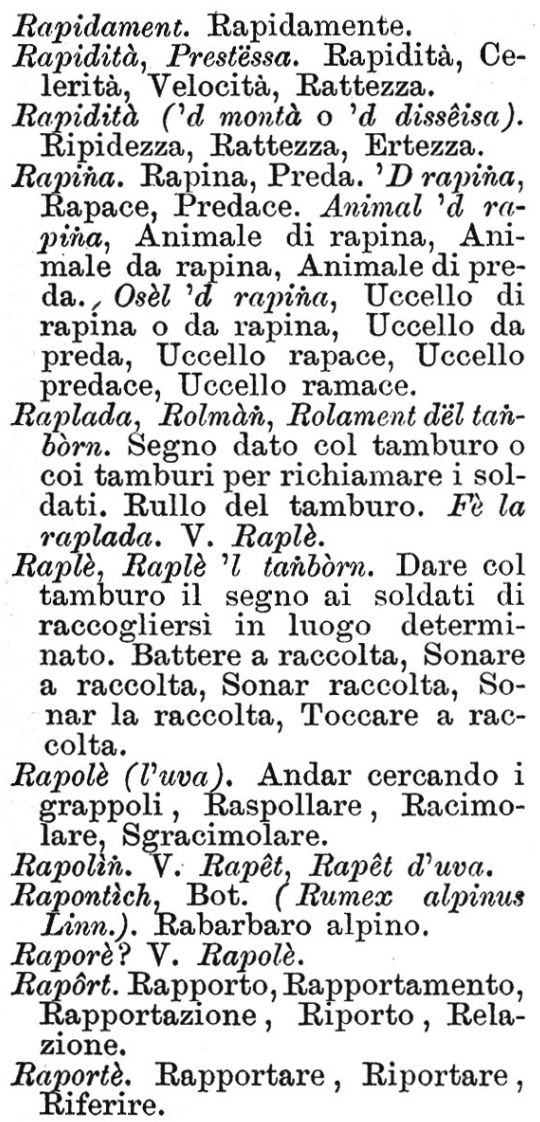

Già il lemmario ${ }^{71}$ non è affatto ridotto rispetto al Sant'Albino, cfr. Sant'Albino (1859: 957b): manca solo rapelura, ma ci sono in più raplada, rapontlich e raporè. Aggiunte che non sono peraltro prive di interesse: rapontlich è termine botanico raro, sconosciuto all'infuori dello Zalli (1814.2: 278b, ovviamente con diversa ampiezza), e raporè è variante probabilmente langarola (se non sbaglio del solo Gavuzzi) di quello che in torinese è rapulè; àmbito, in entrambi i casi, più del contado che della corte.

Ma è anche all'interno delle voci che si coglie la differenza: un confronto con le zone corrispondenti del Sant'Albino può essere illuminante: si vedano due campioni dalle pp. 975b e 975b. Qui le zone fraseologiche e non "urbane" sono ampliate, come ben si vede da rapina = rapina, dove la locuzione osèl d' rapina è aggiunta ma il materiale esplicativo è praticamente identico, solo che nel Sant'Albino è finalizzato più all'italiano che al piemontese ${ }^{72}$. Diversamente va per gli altri àmbiti, dove il Sant'Albino dispiega tutta la sua raffinatezza, e per le zone più marcatamente grammaticali; si confrontino le voci rapôrt e raport.

71 È semmai l'assetto delle voci ad essere più compatto.

72 A volte però le cose vanno diversamente: ad esempio la voce popò 'upupa', Gavuzzi (1891: 483a) = Sant'Albino (1859: 914a), è più sviluppata nel Sant'Albino, che registra anche la forma langarola putta. 
Raport. Rapporto, riporto, relazione, ragguaglio.

- Rapporto, riporto (T. d'arit.). II numero che si porta da una colonna all'altra nelle addizioni o ne' sommati.

n- Correlazione, attenenza, connessione, congruenza, dependenza, convenienza, rassomiglianza; 0 sempl. conoscenza, amicizia.

- Nel num. del più, presso alcuni artefici, que' pezzi che si adattano per ornamento a qualche lavoro. Pess draport. Rapporti.

- d'parentela, d'amicissia. Relazione, attenenza di parentela, d'amicizia fra due o più persone.

Rapert a..... avv. Quanto a, rispetto a, riguardo a..... Raport a col afè. Rispetto, quanto a quell'affare.
Rapina. Rapina. Il torre altrui checchessia con violenza. Dicesi anche per la cosa rapita; altr. preda.

- Animal d'rapina. Animale di o da rapina o di preda. Quello che rapisce gii altri per ciharsene. Parlandosi di volatili, dicesi più comun. uccello ramace (perchè tali uccelli errano di ramo in ramo); benchè si dica anche rapace $o$ di preda.

Già da questi pochi campioni dovrebbero emergere i non pochi elementi di originalità del Gavuzzi: lessicograficamente, maggiore attenzione alla fraseologia (che diventerà il cavallo di battaglia dei Viriglio), meno all'assetto grammaticale e più cura delle varianti dei "dialetti" del piemontese diversi dalla "lingua" torinese ${ }^{73}$, che come tale è ormai decaduta dal suo ruolo.

Quello che è più cospicuo è infatti il diverso rapporto che si configura tra piemontese ed italiano; come diremo per la fase successiva, le danze sono state avviate decisamente col Ponza, ed i giochi sono ormai fatti col Sant'Albino: al piemontese è stato irrimediabilmente definito un ruolo subalterno rispetto all'italiano. Se ad assumerne completamente tutte le conseguenze sarà la generazione successiva, già il Gavuzzi ne ha il pieno sentore ed elabora una strategia diversa ${ }^{74}$ dal Sant'Albino, riportando il piemontese al centro dell'attenzione, e non solo il "piemontese illustre", ma anche quello del contado.

E questo è confermato anche dall'altra grande innovazione del Gavuzzi: avere creato un vero dizionario italiano - piemontese, che non fosse solo l'effetto del rovesciamento di un dizionario piemontese - italiano, perlopiù fortemente abbreviato, poco più di un indice inverso ${ }^{75}$ : che tali sostanzialmente erano le prove in tal senso del Ponza, la cui natura ancillare era peraltro esplicita.

A riprova, il vocabolario è anche accompagnato da una grammatica (il Trattatello, pp. 3-58) di ben diversa consistenza di quelle del Ponza, che non doveva servire ad altro che a meglio imparare l'italiano.

73 Questo atteggiamento è fin esplicitato (anche se in modo forse non chiarissimo) nell' «Avvertenza», dove programmaticamente si dichiara che «un vocabolario piemontese che non registrasse che le voci poco o nulla dissimili dall'italiano, come Fratèl, Sorèla, Aqua, Vëdi, Goardè, Conprè, ed omettesse le corrispondenti, vere dialettali, Frèl, Seurî, Vòghi, Beichè, Buchè, Catè, sarebbe pressoché inutile: se poi queste ultime avesse per basse, farebbe offesa immeritata agli abitanti del contado o dei comunelli rurali, dove tali voci sono tuttora in onore, ed allora, addio dialetto. Questo Vocabolario adunque, essendo regionale, non deve avere e non ha tali distinzioni, [...]», Gavuzzi (1891: v). Strategia, peraltro, completamente in antitesi a quella del Pasquali che, insipienza etimologica a parte, aveva per scopo proprio «mettere in risalto 1" italianità del dialetto piemontese" [Pasquali (1869: ix)], principio questo che da solo comporta una selezione del materiale nella direzione dell'abbandono dei termini più caratteristici e a vantaggio di una preponderanza degli italianismi» (L. Ramello 2004: 51).

74 Nella Premessa all'italiano - piemontese Gavuzzi (1896: v-vi) accampa, è vero, le consuete giustificazioni didattiche, ma il tono della ripetizione suona diverso, ed è in qualche modo operazione di parata: si veda (e gusti) l'aperta derisione di tanto italocentrismo (che noi posteri possiamo ben apprezzare più dei suoi contemporanei) nell'osservazione che il governo «spende 30 mila lire pel Vocabolario del dialetto toscano e 40 mila lire annue per quello della famosa Crusca, che secondo i migliori calcoli non sarà terminato che nel 1973» (1896: vii).

75 Ha uguali dimensioni e numero di pagine del suo compagno, ma ha ben 50.000 voci, che sono quindi mediamente assai brevi (a volte solo col traducente secco). 


\section{Epilegomena}

Dopo la codificazione del Sant'Albino con gli addenda del Gavuzzi, un'ultima cosa notevole va segnalata, che ci porta un poco fuori del periodo su cui ci siamo concentrati, ossia dalla fine dell'Ottocento ai primi del Novecento: a fine secolo, infatti, a giochi ormai fatti, nasce una letteratura "memorialistica", aneddotica ed in pantofole, per così dire, che ha anche notevole interesse lessicografico ${ }^{76}$, e che è ormai specificamente volta a documentare il piemontese, il cui destino è percepito come ormai segnato.

\subsection{Il Viriglio}

Il nome cui il fenomeno è particolarmente legato è quello di Alberto Viriglio ${ }^{77}$, nella cui opera, peraltro letterariamente godibilissima ${ }^{78}$, sono date a piene mani locuzioni (campo in cui già eccelleva il Ponza, come abbiamo visto), paremiologie (di cui v'erano interessanti precedenti nel Capello) ed altre espressioni rare o di rilevante interesse storico-antropologico. Il tutto associato a capacità definitorie abbastanza eccezionali, paragonabili per efficacia al Fanfani: come non ricordare sue definizioni come «Magòné $e^{79}$ - Affliggersi in silenzio», Viriglio (1897: 30), o «Fôsônè - Riescire utilizzato abbondantemente» (1897: 29) o «Gariè - Scavare grattando» (1897: 29).

Un piccolo esempio da Viriglio (1897: 14), illustrativo del tipo di operazione da aspettarsi, in cui è peraltro da notare che se pure tutti i lemmi di base sono nel Sant'Albino, solo tre delle fraseologie addotte lo sono (rispettivamente 860b, 718b e 275a): quelle dei cenci, dei gigli e delle braci, forse perché c'erano corrispondenti toscani.

È raro che occorra nel discorso un aggettivo senza che si presenti immediatamente un «come» per illustrarlo.

\begin{tabular}{|c|c|c|}
\hline Bianc & côme & una pata. \\
\hline Candi & $\gg$ & un liri. \\
\hline$\gg$ & $\gg$ & n'agnel. \\
\hline Neir & $\gg$ & un singher. \\
\hline$\gg$ & $\gg$ & un capel. \\
\hline$》$ & $»$ & un crôass. \\
\hline$\gg$ & $\gg$ & 'l tabarô dël Diâ̂. \\
\hline Rôss & $»$ & un pito. \\
\hline$\gg$ & $»$ & la brasa. \\
\hline Bianc e rôss & $\gg$ & una fiôr. \\
\hline
\end{tabular}

76 In verità vi è anche un dizionario scolastico, Salamitto CB 3150, che mi risulta solo alla biblioteca civica di Mondovì (FL.D.25.1.Salag.1), stampato, appunto, a Mondovì nel 1893. Non ho potuto vederlo, ma presentandosi «ad uso delle scuole elementari, serali e festive del Piemonte», ed essendo di sole 89 pagine, presumo che sia solo un'opera "di servizio" come i Dizionarietti del Ponza, e che, per quanto attardata, andrà collocata ancora in quel solco.

77 «Arguto poeta dialettale | de l'antica vita torinese | indagatore e storiografo | di glorie e memorie paesane | geniale evocatore», secondo lo commemora la lapide posta a Torino in Piazza Palazzo di Città, accanto al municipio. Linguisticamente, è ricordato soprattutto per avere introdotto un'ortografia che ha goduto di una notevole diffusione (dal nome di lui è di solito detta appunto virigliana) prima dell'affermazione dello standard moderno.

78 Il tono dell'autore, tra l'arguto e l'erudito, ben si può immaginare dal noto detto scolastico, in latino maccheronico, Gratatio capitis facit recordare cosellas, che Viriglio pone in epigrafe ai suoi Scandagli del 1897.

79 Mantengo qui e nell'esempio successivo l'ortografia caratteristica dell'autore; a norma moderna sarebbero rispettivamente magoné, fosoné e garié. 


\subsection{Il Comune ed il Rosa}

Questa "linea aneddotica" sopravvisse all'autore almeno fino a Comune (1923), non privo di interesse, anche se certo non all'altezza del Viriglio; che tuttavia qualche antecedente pur l'aveva. Penso soprattutto al Glossario dell'89 del Rosa: attrezzato glottologo e linguista storico, studioso di toponomastica ed etimologia, il Rosa pur partendo evidentemente da quei domini, a lui consueti, si avvicina molto a quella che sarà la formula del Viriglio.

\subsection{D'Azeglio}

Ma anche gli Studi di un ignorante di Emanuele D'Azeglio potrebbero costituire parzialmente un precedente in questo senso. E dico parzialmente perché gli Studi (come d'altro canto il titolo stesso lascia immaginare) non sono monocordi: la prima parte (pp. 7-47), le «Parole prette piemontesi | alle quali non corrisponde in italiano la parola identica», anzi, costituiscono forse la prima eccezione lessicografica per cui esplicitamente non tanto l'italiano, ma il piemontese stesso, sia il fine; le parti che seguono, cioè $\mathrm{i}$ «Modi di dire piemontesi | che non sempre sarebbero intesi da altri italiani» (pp. 47-90) ed i «Proverbi piemontesi» (pp. 91-125) lo sono di più; ma, tolte le tre brevi sezioni «Parole piemontesi | derivate dall'italiano ma modificate» (pp. 126-128), «Parole piemontesi | derivate dal francese» (pp. 129134) e «Frasi latine | che si usano non di rado parlando piemontese» (pp. 135-141), sono soprattutto le due successive «Parole antiquate e fuori d'uso» (pp. 142-151), pur desunte principalmente (e dichiaratamente) da Sant'Albino, Zalli e Pipino, e ancor più i «Nomi di stoffe | e di articoli di moda e di vestiario del secolo scorso» (pp. 152-156) a ricondurci a quella vena garbatamente nostalgica che sarà del Viriglio (quello che ne manca, anche qui, è però il tono aneddotico); le sezioni finali, cioè gli «Esempi di piemontese antico» (pp. 157-181), i «Dialetti piemontesi» (pp. 182-186), «Provenzale» (pp. 187-190) nonché la ripresa di quei «Voci e modi toscani $\mid$ raccolti da | Vittorio Alfieri | con le corrispondenze dei medesimi in lingua francese $\mid \mathrm{e}$ in dialetto piemontese» che avevamo visto già editi dal Cibrario nel 1827 , inclinano più sulla semplice erudizione che altro.

\section{Conclusioni}

Questa prima disamina intendeva solo fornire le linee portanti della tradizione lessicografica piemontese, da cui già emergono elementi di rilevanza; anche se una migliore caratterizzazione lessicografica, per ragioni di spazio, sarà consegnata ad altri interventi, in buona parte peraltro già scritti.

\section{Appendice: i testi}

Nell'intento di fornire, per il piemontese, un repertorio il più possibile esauriente $\mathrm{e}$ di riferimento, sganciato da semplici necessità bibliografiche, riproduco come appendice $\mathrm{i}$ frontespizi in modo quasi integrale, dandone pure la paginazione, e quando v'è, la numerazione della Bibliografia dei Clivio. 
Alfieri

1827

Capello

1814

Comune

1923

dal Pozzo

1888

D’Azeglio

1886

Gavuzzi

1891

Voci $\mid$ e $\mid$ modi toscani $\mid$ raccolti $\mid$ da Vittorio Alfieri | con le corrispondenze de' medesimi | in lingua francese | ed in dialetto piemontese | Torino | per l'Alliana. A spese di P. G. Pic | librajo della R. Accademia delle scienze | MDCCCXXVII; pp. 48. CB 3126.

Dictionnaire | portatif | piémontais - français | suivi |d'un vocabulaire français | Des termes usités dans les arts et métiers, par ordre | alphabétique et de matières, avec leur explication | par Louis Capello | comte de Sanfranco | [...] | Turin, de l'Imprimerie de Vincent Bianco | 1814, due tomi. I I. Pièmontais-français, e II. Français; pp. risp. XII+560+20 e 950. CB 3120 .

Vocaboli dialettali piemontesi | d'un tempo | (1400-1800) | raccolti e commentati con cenni storici | e curiosità etimologiche $\mid$ dal $\mid \mathrm{Comm}^{\text {re }}$ Francesco Emilio Comune Cav. Maur. ${ }^{\text {no }}$ | di Asti (dall'Astese G. G. Alione all'Astese A. Brofferio) $|[\ldots]|$ Sanremo, Tipografia Conti \& Gandolfi, Via Roma, 16 | 1923. CB 3151.

Maggiore dal Pozzo | Glossario etimologico | piemontese | Torino | F. Casanova, Libraio-Editore | 1888; pp. 250. CB 3176.

E[manuele] D’Azeglio | Studi di un ignorante $\mid$ sul | dialetto piemontese | Torino | Unione tipografico-editrice | 1886. CB 3053, 3247.

1896

1869

Vocabolario | piemontese - italiano | compilato | da | Giuseppe Gavuzzi da Caramagna | ingegnere giubilato degli Uffici tecnici di Finanza | [...] | 1891 | L. C. Roux e C. - Editori | Torino - Roma; pp. XII+692. CB 3148. Giuseppe Gavuzzi | Vocabolario | italiano - piemontese | 1896 | Torino | Tipografia Fratelli Canonica; pp. VIII+696. CB 3117.

Nuovo | dizionario | piemontese-italiano | ragionato e comparato alla lingua comune | coll'etimologia di molti idiotismi | premesse alcune nozioni filologiche sul dialetto | del Prof. | Giovanni [Battista] Pasquali | Torino | 1869 | Libreria editrice di Antonio Moreno. Anche anastatica: Ann Arbor, University of Michigan Library, [s.d.]. CB 3146 (stesso numero della seconda edizione).

1870 Nuovo | dizionario | piemontese-italiano | ragionato e comparato alla lingua comune | coll'etimologia di molti idiotismi | premesse alcune nozioni filologiche sul dialetto | del Prof. | Giovanni [Battista] Pasquali | Seconda edizione | Torino | 1870 | Libreria editrice di Antonio Moreno. Anche anastatica: Bologna, Arnaldo Forni Editore, 1997. CB p. 193a: «identica alla prima»; CB 3146.

Pipino

$1783 \quad$ Vocabolario | piemontese | del medico | Maurizio Pipino | A sua Altezza Reale | Maria Adelaide | Clotilde Saveria | di Francia | Principessa di Piemonte | Torino, nella Reale Stamparia | M.DCC.LXXX.III, pp. $\mathrm{X}+96+[12]+97-226 . \mathrm{CB} 3122$. 
Ponza

1826 Dizionario piemontese - italiano, volume secondo dell'Inviamento al comporre nella lingua italiana, approvato dalla R. Direzione delle Scuole e seguito da un dizionario piemontese-italiano, Torino, vedova Ghiringhello, 1826. CB 3129.

1827 Dizionario piemontese - italiano approvato dalla R. Direzione delle Scuole, Torino, vedova Ghiringhello, 1827; non visto, pp. 112. CB 3130.

1830-33 Vocabolario | piemontese - italiano | di | Michele Ponza | da Cavour, Torino $\mid[\ldots]$ dalla Stamperia Reale, tre volumi. q Vol. I. $A-D$, pp. XXIII+440, 1830; Vol. II. DP, pp. 348, 1832, Vol. III. $Q-Z, 1833$, pp. 212. CB 3131.

1834 Dizionarietto | piemontese - italiano | contenente | le voci puramente piemontesi | e di uso famigliare e domestico | del Sac. M[ichele] Ponza | terza edizione corretta ed ampliata | Torino | dalla Stamperia Reale | 1834; pp. 165. CB 3137.

1838 Donato $\mid$ piemontese - italiano $\mid$ ossia $\mid$ manuale $\mid$ della lingua italiana $\mid$ ad uso | de' maestri e degli scolari piemontesi | di | Michele Ponza | Torino, 1838, | dalla Tipografia Baggione, Melanotte e Pomba; la parte seconda (pp. 97-156) è costituita da Temi piemontesi da tradursi in italiano | per esercizio pratico di lingua; segue un Vocabolarietto piemontese ed italiano | di alcune voci piemontesi di più difficile traduzione, | contenute nei temi precedenti (pp. 137-140). CB 3265, 217, 3087.

1843 Vocabolario | piemontese ital. e italiano - piem. | del Sac. Michele Ponza | maestro giubilato di grammatica italiana e latina | visitatore delle scuole della provincia di Torino | pensionario del Real ordine civile di Savoja $|[\ldots]|$ Torino, 1843 | Tipografia di Gio. Battista Paravia e Comp.; pp. II+140+64. CB 3138. Vocabolario | piemontese - italiano e Vocabolario | Italiano - Piemontese con paginazioni indipendenti.

1844 Vocabolario | piemontese - italiano | del Sac. Michele Ponza | maestro giubilato di grammatica italiana e latina | visitatore delle scuole della provincia di Torino | pensionario del Real ordine civile di Savoja $|[\ldots]|$ Volumi due ${ }^{80}[\ldots]|[\ldots]|$ Torino, 1844 | Stamperia Ferrero, Vertamy e Comp.; seguito da un'Appendice contenente (con paginazioni autonome) una Teorica dei nomi e dei verbi italiani, una Appendice al vocabolario piemontese-italiano, una Aggiunta di voci e frasi piemontesi - italiane, e chiuso da una Tavola delle abbreviature, ed una Tavola delle correzioni a farsi (con numerazione romana); pp. II+130+2+$\mathrm{VI}+14+72+34+2+\mathrm{V}$. CB $3140^{81}$.

1846 Vocabolario | piemontese - italiano $|e|$ italiano - piemontese $\mid$ del sacerdote Michele Ponza | maestro giubilato di grammatica italiana e latina, | pensionario del Real ordine civile di Savoja, ecc., | [...] | Edizione terza | accresciuta di circa 2000 voci principalmente d'uso domestico | Torino | presso Carlo Schiepatti librajo-editore, | [...] | 1846; pp. XVI+769; preceduto da una «Teorica dei nomi e dei verbi italiani» (pp. 1-32) e da una

80 In realtà tutti gli esemplari che ho visto si presentano in un volume unico; probabilmente la dizione si riferirà alla cospicua presenza dell'Appendice, tanto più notabile se la pubblicazione, come d'uso, è avvenuta a fascicoli.

81 La sua paginazione però non corrisponde perfettamente alla realtà, né tantomeno la divisione in volumi. 
1847 Vocabolario $\mid$ piemontese-italiano $\mid$ e $\mid$ italiano - piemontese $\mid$ del sacerdote Michele Ponza | maestro giubilato di grammatica italiana e latina, | pensionario del Real ordine civile di Savoja, ecc., | [...] Edizione quarta | accresciuta di circa 1200 voci principalmente d'uso domestico | Torino | presso Carlo Schiepatti librajo-editore, | [...] | 1847; pp. XVI+769; preceduto da una «Teorica dei nomi e dei verbi italiani» (pp. 1-32) e da una «Teorica di gramatica piemontese - italiana» (pp. 33-47) comprende un «Vocabolario - piemontese - italiano» (pp. 53-591) ed un «Vocabolario | Italiano - Piemontese» (593-769) che sostanzialmente riprende, con poche modifiche, quello del '43. CB 3141.

1859 Vocabolario | piemontese - italiano $\mid$ del professore $\mid$ di gramatica italiana e latina $\mid$ Michele Ponza | Edizione quinta | corretta ed accresciuta di circa il doppio | delle voci $\mid$ Pinerolo | dallo Stabilimento tipografico-librario | di Giuseppe Lobetti-Bodoni | 1859; pp. 863. CB 3142R (che lo dichiara erroneamente «edizione sesta» scambiandolo con quello del '60 che, di fatto, è identico).

1860 Vocabolario | piemontese - italiano | del professore | di gramatica italiana e latina $\mid$ Michele Ponza $\mid$ Edizione sesta | corretta ed accresciuta di circa il doppio | delle voci $\mid$ Pinerolo | dallo Stabilimento tipografico-librario | di Giuseppe Lobetti-Bodoni, 1860, pp. 863. CB vacat.

Rosa

1883

L'elemento tedesco $\mid$ nel $\mid$ dialetto piemontese $\mid$ Postille etimologiche $\mid$ di | Ugo Rosa | Torino | Vincenzo Bona | 1883; pp. 29. CB 3179

1889 Ugo Rosa | Glossario storico popolare | piemontese | Dichiarazione di CCX voci, motti locali e locuzioni proverbiali $\mid$ di origine storica $\mid$ Torino, Libreria Ermanno Loescher di Carlo Clausen | 1889 | [...]; pp 118. CB 3184.

Sant'Albino

1859 Gran $\mid$ dizionario | piemontese - italiano | compilato | dal cavaliere | Vittorio di Sant'Albino | Torino | Società l'Unione Tipografico-Editrice Torinese | 1859; pp. XVI+1237. CB 3145. Anche anastatiche: a cura di Corrado Grassi, Torino, Bottega d'Erasmo, 1964; a cura di Giuliano Gasca Queirazza, Savigliano, L'artistica di Savigliano, 1893.

Viriglio

1897

1917

Vopisco

1564
Alberto Viriglio | Come si parla $\mid$ a Torino | Impressioni e scandagli $\mid$ $[\ldots] \mid$ Torino $\mid$ S. Lattes \& C. — Libreria scientifico-letteraria $|[\ldots]|$ 1897; pp. 62. CB 3054, 3252, 3308, 3318.

Alberto Viriglio | Voci e cose $\mid$ del $\mid$ vecchio Piemonte | Torino | S. Lattes \& C., Editori | Librai della Real Casa | 1917; pp. 313. CB 3055.

Michaelis | Vopisci | Neapolitani | humaniorum litteraru(m) in vtraq(ue) | lingua professoris | Promptuarium. | Additamentum eiusdem de Calendis, Vt $\mid$ facillimum, ita maxime utile. | In Ducali Typographia Montis Regalis, apud | Leonardum Torrentinu(m), MDXLIII. Edizione anastati- 
ca: Il promptuarium: vocabolario volgare - latino di Michele Vopisco, Mondovi 1564, presentazione di Giuliano Gasca Queirazza, Torino, Bottega d'Erasmo, 1972. CB 3118.

Zalli

1815 Disionari piemontèis, italian, latin $\mid$ e fransèis $\mid$ conpöst $\mid$ dal preive Casimiro Zalli $\mid$ d'Cher $|[\ldots]|$ Carmagnola $|1815|$ da la Stanparia d'Peder Barbiè, tre tomi. ๆ I. $A$-C, pp. 508, II. I-S, pp. 496, III. $S$-Z, pp. 606. CB 3123.

1830 Dizionario | piemontese | italiano, latino e francese | compilato | dal Sac. Casimiro Zalli | di Chieri | edizione seconda | riordinata e di nuovi vocaboli arricchita $|[\ldots]|$ Carmagnola, dalla Tipografia di Pietro Barbiè | 1830, due volumi. ๆ Vol. I. $A-L+$ «Appendice» | al primo volume | colla $\mid$ tavola delle abbreviature $\mid$ usate $\mid$ in questa ristampa, pp. VIII $+498+115+\mathrm{V}$; Vol. II. $M-Z+$ «Appendice» | al tomo secondo, pp. 620+38+VI-IX. CB 3124.

\section{Riferimenti bibliografici e abbreviazioni}

Aa. Vv. (1612): Vocabolario degli Accademici della Crusca con tre indici delle voci, Venezia, Giovanni Alberti. [Ristampa anastatica, con «Presentazione» di Giovanni Nencioni, Firenze, Le Lettere, 1987. Abbreviato Prima Crusca ${ }^{82}$.

Aa. Vv. (1623): Vocabolario degli Accademici della Crusca, in questa seconda impressione da' medesimi riveduto, e ampliato, Venezia, Iacopo Sarzina. Abbreviato Seconda Crusca.

Aa. Vv. (1691): Vocabolario degli Accademici della Crusca, in questa terza impressione nuovamente corretto, e copiosamente accresciuto, Firenze, Stamperia dell'Accademia della Crusca, tre volumi. ๆ Vol. I. [«Premesse», «Indici» e «Giunte»]; Vol. II. AI; Vol. III. LZ. Abbreviato Terza Crusca.

Aa. Vv. (1729-31): Vocabolario degli Accademici della Crusca, quarta impressione, Firenze, Domenico Maria Manni, 6 volumi. ๆ Vol. I. A-C, 1729; vol. II. D-I; vol. III. L-P; vol. IV. $Q-S$; vol. V. T-Z; vol. VI. «Autori», «Tavole» ed «Indici», 1738. Abbreviato Quarta Crusca.

Aa. Vv. (1741): Vocabolario degli Accademici della Crusca, quinta impressione, Venezia, Francesco Pitteri. ๆ Vol. I. $A$-C; vol. II. D-I; vol. III. $L-P$; vol. IV. $Q-S$; vol. V. T-Z. Abbreviato Crusca veneziana.

Aa. Vv. (1806): Vocabolario degli Accademici della Crusca, Verona, 1806, Stamperia di Dionigi Ramanzini. ๆ Vol. I. $A-B$; vol. II. $C-D$; vol. III. E-I; vol. IV. $K$ - $O$; vol. V. P-R; vol. VI. $S$ - $T$; vol. VII. $U$-Z. Abbreviato Crusca veronese.

Aa. Vv. (1841-51): Nuova enciclopedia popolare, ovvero dizionario generale di scienze, lettere, arti, storia, geografia, ecc., ecc, Torino, Giuseppe Pomba e Comp. Editore. $\uparrow$ Vol. I. $A$, 1841; Vol. II. $B, 1842$; Vol. III. $C$, 1845; Vol. IV. $C D, 1848$; Vol. V. $E F, 1845$; Vol. VI. GH, 1846; Vol. VII. IK, 1847; Vol. VIII. LM, 1847; Vol. IX. MN, 1847; Vol. X. OP, 1848; Vol. XI. RS, 1849; Vol. XII. TZ, 1848; Vol. XIII. Supp, 1851.

Alberti di Villanuova, [Francesco] D' (1797-1805): Dizionario universale critico, enciclopedico della lingua italiana, Lucca, Stamperia di Domenico Marescandoli. ๆ Vol. I. $A-C a$,

82 Tutte le prime quattro (e parzialmente la quinta) Crusche sono ormai interrogabili online dal sito mantenuto dall'Accademia della Crusca. 
1797; Vol. II. Ce-E, 1797; Vol. III. F-I, 1798; Vol. IV. K-O, 1803; Vol. V. P-R, 1804; Vol. VI. $S-Z, 1805$.

Alberti di Villanuova, [Francesco] D' (1825): Dizionario universale critico, enciclopedico della lingua italiana, riveduto e corretto, seconda edizione e prima milanese, Milano, Luigi Cairo. 1 Stessa divisione in volumi.

Alfieri, Vittorio (1951a): Vita scritta da esso, volume I, edizione critica della stesura definitiva a cura di Luigi Fassò, Asti, Casa d'Alfieri, "Opere di Vittorio Alfieri da Asti” 1.

Alfieri, Vittorio (1951a): Vita scritta da esso, volume II, prima redazione inedita della Vita, Giornali, Annali e documenti autobiografici, edizione critica a cura di Luigi Fassò, Asti, Casa d'Alfieri, "Opere di Vittorio Alfieri da Asti” 2.

Aly Belfàdel, Arturo (1907): Grammatica magiara, con esercizi e vocabolarietto, Milano, Ulrico Hoepli, "Manuali Hoepli".

Âly-Belfàdel, Arturo (1933): Grammatica piemontese, Noale (Venezia), Tipografia - Cartoleria L. Guin.

Barbera, Manuel (i.c.s.): «Michele Ponza lessicografo, didatta e purista», Festschrift per Salvatore Claudio Sgroi.

Barbiere, mastro Simone (1831): Osservazioni sopra l'Annotatore degli errori di lingua, Torino, Cassone, Marzorati e Vercelloti.

Beccaria, Gian Luigi / Soletti, Elisabetta, (edd.) (2005): La lessicografia a Torino dal Tommaseo al Battaglia. Atti del Convegno per il bicentenario della nascita di Niccolò Tommaseo (Torino-Vercelli, 7-9 novembre 2002), Alessandria, Edizioni dell'Orso, "In forma di parola".

Bellone, Luca / Cura Curà, Giulio / Cursietti, Mauro / Milani, Matteo (edd.) (2002): Filologia e linguistica. Studi in onore di Anna Cornagliotti, Alessandria, Edizioni dell'Orso, 2012.

Bianco, Alessandro (2003): «Vittorio Righini di Sant'Albino (1787-1865): la fortuna di un filologo dilettante», Studi piemontesi, XXXII, pp. 449-61.

Biondelli, B[ernardino] (1853): Saggio sui dialetti gallo-italici, Milano, Gius. Bernardoni di Gio.

Boerio, Giuseppe (1829): Dizionario del dialetto veneziano, Venezia, Andrea Santini e figlio.

Boerio, Giuseppe (1829): Dizionario del dialetto veneziano, seconda edizione aumentata e corretta, aggiuntovi «l'Indice italiano - veneto» già promesso dall'autore nella prima edizione, Venezia, Giovanni Cecchini edit[.].

Boerio, Giuseppe (1829): Dizionario del dialetto veneziano, terza edizione aumentata e corretta, aggiuntovi «l'Indice italiano - veneto», Venezia, Giovanni Cecchini edit.

Burdet, Carlo A[lfonso] M[aria] (2005): «Il medico Maurizio Pipino (1739-1788). Cenni biografici con due sue lettere inedite», in Almanacco piemontese | Armanach piemontèis, XXXV, pp. 161-170.

Capello comte de Sanfranco, Louis (1833): Dictionnaire de mythologie de tous les peuples avec les rapprochements historiques, Turin, Imprimerie de Joseph Favale.

Cardinali, Francesco (1827-28): Dizionario portatile della lingua italiana, Bologna, Tipografia di Iacopo Marsigli; due volumi. ๆ Vol. I. [A-inspiratóre], 1827; Vol. II. [L-zurro], 1828.

Cardinali, Francesco / Borrelli, Pasquale (1846-51): Dizionario della lingua italiana già pubblicato da Francesco Cardinali e novellamente corretto nelle dichiarazioni de' vocaboli, aumentato di spiegazioni etimologiche e di vocaboli omessi, ed in più altre guise migliorato dall'avvocato Pasquale Borrelli, Napoli, Gaetano Nobile Editore-Proprietario; tre volumi. ๆ I. [A - gutturalmente], 1846; II. [H - puzzura], 1846; III. [Q - zurro], 1851. 
Carena $^{83}$, Giacinto (1840): Prontuario di vocaboli attenenti a parecchie arti, ad alcuni mestieri, a cose domestiche e altre di uso comune per saggio di un Vocabolario metodico della lingua italiana, Firenze, Galileiana.

Carena, Giacinto (1846): Prontuario di vocaboli attenenti a parecchie arti, ad alcuni mestieri, a cose domestiche e altre di uso comune per saggio di un Vocabolario metodico della lingua italiana, parte prima, Vocabolario domestico, Torino, Stabilimento tipografico Fontana.

Carena, Giacinto (1853): Prontuario di vocaboli attenenti a parecchie arti, ad alcuni mestieri, a cose domestiche e altre di uso comune per saggio di un Vocabolario metodico della lingua italiana, parte seconda, Vocabolario metodico d'arti e mestieri, Torino, Stamperia Reale.

$\mathrm{CB}=$ Clivio - Clivio (1971)

Cherubini, Francesco (1839-56): Vocabolario milanese - italiano, Milano, Imp. Regia Stamperia - Società Tipografica de' Classici Italiani, cinque volumi. ๆ Vol. I. $A-C$, dall'Imp. Regia Stamperia, 1839; Vol. II. D-L, dall'Imp. Regia Stamperia, 1840; Vol. III. $M-Q$, dall'Imp. Regia Stamperia, 1841; Vol. IV. $R-Z+$ «Giunte e correzioni», Imp. Regia Stamperia, 1843; Vol. V. «Sopraggiunta», dalla Società Tipografica de’ Classici Italiani, 1856.

Clivio, Amedeo / Clivio, Gianrenzo P. (1971): Bibliografia ragionata della lingua regionale e dei dialetti del Piemonte e della Valle d'Aosta, e della letteratura in piemontese, Torino, Centro studi piemontesi | Ca dë studi piemontèis. Abbreviato CB.

Cornagliotti, Anna (1991): «I francesismi nel "Vocabolario Piemontese” di Maurizio Pipino (1783)», Studi piemontesi, XX, pp. 313-20.

Cornagliotti, Anna (2002): «Un vocabolario piemontese dimenticato: il "Dissiunari Piemuntèis" di Gioacchino Brovardi», Studi piemontesi, XXXI, pp. 341-52.

Cornagliotti, Anna (2015): Repertorio etimologico piemontese (REP), redattori Luca Bellone, Anna Cerutti Garlanda, Anna Cornagliotti, Marisa Falconi, Laura Parnigoni, Giovanni Ronco, Consolina Vigliero, Torino, Centro studi piemontesi | Ca dë studi piemontèis.

[Costa, Paolo / Cardinali, Francesco] ${ }^{84}$ (1819-26): Dizionario della lingua italiana, Bologna, fratelli Masi e comp. [ma VII: Riccardo Masi]; sette volumi. ๆ Vol. I. $A, 1819$; Vol. II. $B C$, 1820; Vol. III. DEF, 1821; Vol. IV. GHIJLM, 1822; Vol. V. NOPQR, 1823; Vol. VI. S, 1824; Vol. VII. TUVXYZ ed «Appendici», 1826.

Crusca veneziana $=\mathrm{AaVv}(1741)$

Crusca veronese $=\mathrm{AaVv}(1806)$

Fanfani, Massimo (2016): «Il dizionario di un'Italia nuova», in UTET: il laboratorio della parola, saggi di Claudio Marazzini, Massimo Fanfani; testimonianze di Tullio de Mauro, Raffaele Simone, Torino, UTET “Grandi opere” 225 = "Strenna Utet 2017”.

Fanfani, Pietro (1863): Vocabolario dell'uso toscano, Firenze, G. Barbèra Editore; due volumi. Anche anastatica, con «Prefazione» di Ghino Ghinassi, Firenze, Casa Editrice Le Lettere, 1976.

Gasca Queirazza, Giuliano (1967), «Presentazione» all'edizione anastatica di Ponza (1859), Torino, Le livre precieux, pp. iii-v.

Gasca Queirazza, Giuliano (1972), «Presentazione» all'edizione anastatica di Vopisco (1564).

83 Non tengo qui conto del rifacimento di Ernesto Sergent del 1877, oltre che per ovvie ragioni cronologiche, perché non è altro che un plagio: cfr. Marello (1980 e 1995: 177). Non considero, inoltre, il terzo volume del Prontuario del Carena perché non è più che un moncherino postumo, troppo frammentario ed incompleto. E non do neppure conto delle tante edizioni che testimoniano della grande fortuna editoriale dell'opera, perché evade dalle finalità di questo contributo.

${ }^{84}$ Menzionati solo in calce alla dedicatoria iniziale Al Cavaliere Vincenzo Monti (I.v-vij). 
Liberatore, Raffaele, diretto da (1829-40): Vocabolario universale italiano compilato a cura della società tipografica Tramater e $C^{i}$., Napoli, Tramater, sette volumi. ๆ Vol. I. A-BU, 1829; Vol. II. C-DU, 1830; Vol. III. E-KU, 1834; Vol. IV. L-O, 1834; Vol. V. P-RU, 1835; Vol. VI. S, 1838; Vol. VII. T-ZU, 1840.

Manuzzi, Giuseppe (1833-40): Vocabolario della lingua italiana, già compilato dagli accademici della Crusca ed ora novamente corretto ed accresciuto, Firenze, David Passigli e socj, due doppi volumi. ๆ Vol. I.1 [A-C], 1833; Vol. I.2 [D-L], 1836; Vol. II.1 [M-R], 1838; Vol. II.2 [S-Z], 1840.

Marazzini, Claudio (1984): Piemonte e Italia. Storia di un confronto linguistico, Torino, Centro studi piemontesi | Ca dë studi piemontèis, "Collana di testi e studi piemontesi" 3.

Marazzini, Claudio (2012): Storia linguistica di Torino, Roma, Carocci, "La lingua delle città italiane".

Marello, C[arla] (1980): Lessico ed educazione popolare. Dizionari italiani metodici dell' '800, Roma, Armando Armando, "Serie di linguistica teorica e applicata" 16.

Marello, Carla (1989): Dizionari bilingui; con schede sui dizionari italiani per francese, inglese, spagnolo, tedesco, Bologna, Zanichelli, "Fenomeni linguistici” 6.

Pacotto, Giuseppe (1967): La letteratura in Piemonte dalle Origini al Risorgimento, documenti e testi scelti e annotati da C[amillo] Brero e R[enzo] Gandolfo, Torino, F. Casanova \& $\mathrm{C}$.

Prima Crusca $=$ AaVv (1612)

Quarta Crusca $=$ AaVv (1729-38)

Ramello, Giuseppe Antonio (1831): Note critiche al primo fascicolo del vocabolario piemontese - italiano di Michele Ponza, Torino, Gio. Battista Paravia Librajo.

Ramello, Laura (2004): «La lessicografia piemontese: profilo storico», Bollettino dell'Atlante linguistico italiano, XXVIII, pp. 27-65.

REP $=$ Cornagliotti (2015)

Massabò, Isabella Ricci (1975): «Capello di San Franco, Luigi», voce in Dizionario biografico degli italiani, Volume 18, Roma, Treccani; online a http://www.treccan.it/enciclopedia/capello-di-san-franco-luigi_(Dizionario-Biografico).

Ronco, Giovanni (2012): «“Il malefico M": beghe tra lessicografi piemontesi», in Bellone et alii (2012), pp. 967-82.

Seconda Crusca $=$ AaVv (1623)

Telmon, Tullio (2015): «La lessicografia dialettale in Piemonte», in Treccani. Lingua Italia$n a, \quad<$ http://www.treccani.it/magazine/ lingua_italiana/speciali/dialetto/Telmon_Diz_ piemont.html>

Terza Crusca $=$ AaVv (1691)

Tommaseo, N[iccolò] (1830): Nuovo dizionario dei sinonimi della lingua italiana, Firenze, Tipografia di Luigi Pezzati.

Tommaseo, Niccolò / Bellini, Bernardo (1865-79): Dizionario della lingua italiana, Torino (ma II.1-IV.1, Torino - Napoli; e IV.2, Pisa - Livorno - Napoli), dalla Società L'Unione Tipografico-Editrice, in sette tomi. $ๆ$ Vol. I.1 $A-A Z Z$, 1865; Vol. I.2 B-CZA, 1865; Vol. II.1 D-FUT, 1869: Vol. II.2 G-LUV, 1869; Vol. III. M-QUO, 1871; Vol. IV.1 R-SVO, 1872; Vol. IV.2 T-ZUZ, «Giunte e correzioni, Tavola delle abbreviazioni», 1879.

Tramater $=$ Liberatore $(1829-40)$

Trissino dal Vello d'Oro, Gian Giorgio (1529): Epistola del Trissino de le lettere nvwvamente

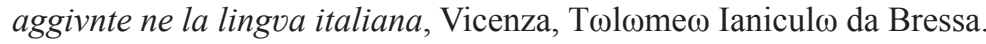

Zecchini, S[tefano] P[ietro] (1848): Dizionario dei sinonimi della lingua italiana, Torino, G. Pomba e C. Editori. 